\title{
Risk of Hyposalivation in Menopausal Patients
}

\author{
Hosseinimehvar $\mathbf{B}^{1^{*}}$, Alaee $\mathbf{A}^{2}$, azizi $\mathbf{A}^{3}$, Shafizade $\mathbf{N}^{4}$ \\ ${ }^{I}$ Student of Dentistry, Oral Medicine Dept, Dental Branch of Tehran, Islamic Azad University, Tehran, Iran \\ ${ }^{2}$ Assistant Professor, Oral medicine Dept, Member of Dental Material Research Center, Dental Branch of Tehran, Islamic Azad University Tehran, Iran \\ ${ }^{3}$ Professor ,Oral Medicine Dept,Dental Branch of Tehran, Islamic Azad University, Tehran, Iran \\ ${ }^{4}$ Assistant Professor, Pediatric dentistry Dept, Dental Branch of Tehran, Islamic Azad University, Tehran, Iran
}

\begin{tabular}{l}
\hline ARTICLE INFO \\
\hline $\begin{array}{l}\text { Article Type } \\
\text { Original Article }\end{array}$ \\
\hline Article History \\
Received: Apr 2017 \\
Accepted: May2017 \\
ePublished: June 2017 \\
\hline
\end{tabular}

Keywords:

Hyposalivation,

Menopause,

Xerostomia \begin{abstract}
Background and aim: Hyposalivation is one of the most common complications during menopause, which affects the quality of life. The aim of the present study was to assess the correlation between menopause and salivary flow rate.

Materials and Methods: This historical-cohort study was conducted on 80 healthy women. Forty postmenopausal women were placed in the case group, while 40 premenopausal women were selected as the control group. The participants were selected from among non-smokers and non-alcoholics and had no systemic diseases. The saliva level of the participants was measured by using the Modified Schirmer's Test (MST) and modified Fox questionnaire. The participants were asked to sit upright, swallow their saliva and hold up their tongue. A strip was placed on the floor of the mouth, and the length of the wet and discolored area on the strip was measured after 3 minutes. Data were analyzed using SPSS 22 according to Mann-U-Whitney and Chi-square tests.

Results: According to the MST, the prevalence of hyposalivation was 35\% in premenopausal women and $65 \%$ in postmenopausal women, with a statistically significant difference between the two groups $(\mathrm{P}<0.001)$. According to the modified Fox questionnaire, the prevalence of normal salivary flow and mild xerostomia in premenopausal women was $90 \%$, while this rate was equal to $62.5 \%$ in postmenopausal women. The prevalence of severe xerostomia was $15 \%$ in postmenopausal women, while none of the premenopausal subjects had severe xerostomia. The incidence of hyposalivation in postmenopausal women is significantly higher than that in premenopausal women $(\mathrm{P}<0.005)$.

Conclusion:It seems that the unstimulated salivary flow rate decreases after menopause.
\end{abstract}




\section{Introduction:}

Hyposalivation is one of the most common complications in dental patients. Many studies have shown that most of these patients are postmenopausal women. ${ }^{(1)}$ The frequency of hyposalivation in postmenopausal women has been reported to be up to $50 \%$, which shows the importance of the correlation between menopause and hyposalivation. ${ }^{(1)}$ A reduction in the salivary flow rate by $40-50 \%$ makes the patient symptomatic and prone to develop xerostomia. ${ }^{(2)}$ Mouth dryness can profoundly affect the quality of life and can interfere with the basic daily functions such as chewing, swallowing and speaking; therefore, it is important to prevent this condition. ${ }^{(2)}$

In a research conducted by Osterberg and colleagues, it was shown that the quantity of saliva significantly decreases in menopausal women. $^{(3)}$

If hyposalivation is left untreated, many problems may occur such as cervical caries, extensive periodontal diseases, oral infections, dysphagia, mucositis and dysgeusia. ${ }^{(4)}$ On the other hand, in some studies no changes were detected in the salivary flow rate of postmenopausal women. ${ }^{(1,4)}$

Therefore, due to the controversies and since hyposalivation can cause oral diseases, the aim of this study was to assess the salivary flow rate in the postmenopausal women referred to the dental branch of Tehran, Islamic Azad University.

\section{Methods and Materials:}

This historical-cohort study was conducted on the women who referred to the oral medicine department of the dental branch of Islamic Azad University of Tehran.

By considering a confidence interval (CI) of $95 \%$, error rate of $5 \%$ and power of $90 \%$, the sample size was calculated to be 40 subjects in each group (case and control). The participants signed written informed consent forms prior to participation in the study. The case group consisted of 40 menopausal women (one-year postmenopause), while the control group consisted of 40 premenopausal women who met the inclusion criteria of the study.

The participants were non-smokers and non- alcoholics with no history of head and neck irradiation. Individuals with systemic diseases such as diabetes mellitus, sarcoidosis, kidney disease, Sjögren syndrome, neurological and thyroid diseases and pregnant women were excluded from the study. ${ }^{(5,6)}$

Assessment of hyposalivation was conducted by using the modified Fox questionnaire according to the severity of the symptoms. ${ }^{(7)}$ The patients were asked to fill the questionnaire. The modified Fox questionnaire includes the following questions:

1. Do you feel you have a dry mouth?

2. Do you need to drink when you are swallowing dry foods?

3. Do you constantly feel thirsty?

4. Do you get stuck in swallowing?

5. Do you feel thirsty all day long?

6. Do you use gum and candy daily to reduce mouth dryness?

Positive responses to the first two questions represent slight mouth dryness, while positive responses to the next two questions represent moderate mouth dryness. Positive responses to the last two questions indicate severe mouth dryness. ${ }^{(7)}$

In this study, the salivary flow rate of the participants was measured by using the Modified Schirmer's Test (MST). This test was done by using sterile test strips (ERC, Turkey) between 9 a.m. and 12 p.m. The patients were prohibited from eating and drinking for 2 hours before the test. After relaxation for a few minutes, the participants were asked to sit upright on the dental unit, swallow their saliva once and hold up their tongue slowly to prevent unintentional wetting of the test strip. Next, the strip was carefully placed on the floor of the mouth by the examiner. After discoloration due to wetting with saliva, the length of the discolored area of the strip was measured 3 minutes later by the examiner. ${ }^{(8-14)}$ After recording the necessary information for data analysis, the results were analyzed using SPSS version 22 software program (IBM Co., Chicago, IL, USA) according to Mann-U-Whitney and Chi-square tests. 


\section{Results:}

Eighty subjects including 40 menopausal women (case group) and 40 premenopausal women (control group) were studied during this research. The mean age of the individuals in the case group was $56.47 \pm 4.43$ years, while the mean age of the participants in the control group was $40.37 \pm 4.78$ years. The salivary flow rate values evaluated by the MST and modified Fox questionnaire are presented in Table 1 categorized by the studied groups.

The MST showed that 14 patients $(35 \%)$ in the control group and 26 patients $(65 \%)$ in the case group had hyposalivation, and Chi-square test demonstrated a statistically significant difference between the two groups $(\mathrm{P}<0.001)$. The MST score was 25.02 in the control group and 19.9 in the case group; the difference between the scores was 5.12 units (20\% lower in the case group), and Mann-UWhitney test showed that this difference is statistically significant $(\mathrm{P}<0.01)$.

According to the modified Fox questionnaire, 36 subjects $(90 \%)$ in the case group and 25 individuals $(62.5 \%)$ in the control group showed no xerostomia or mild xerostomia; the difference between the two groups was statistically significant $(\mathrm{P}<0.001)$. In the control group, none of the subjects showed severe xerostomia; however, in the case group (postmenopausal women) 6 subjects $(15 \%)$ showed severe xerostomia.

Distribution of the individuals based on the salivary flow rate and separated by menopausal status shown in Table 1 demonstrates that according to the modified Fox questionnaire, $10 \%$ of premenopausal women had a low or extremely low salivary flow rate, while $37.5 \%$ of postmenopausal women had a low or extremely low salivary flow rate; Chi-square test showed that this difference was statistically significant $(\mathrm{P}<0.001)$. In premenopausal women, the risk of hyposalivation is $27.5(\mathrm{AR}=25.5)$, while during menopause, the risk of hyposalivation increases to 3.7 times higher $(\mathrm{RR}=3.7)$. The rate of hyposalivation is significantly higher in menopausal women in comparison with that in premenopausal women $(\mathrm{P}<0.005)$.

Table 1: Distribution of the subjects according to the salivary flow rate estimation methods and categorized by the menopausal status

\begin{tabular}{|c|c|c|c|c|c|c|c|}
\hline \multirow[t]{4}{*}{ Salivary flow rate } & \multicolumn{3}{|c|}{ Modified Schirmer's Test (MST) } & \multicolumn{4}{|c|}{ Modified Fox Questionnaire } \\
\hline & \multirow{2}{*}{\multicolumn{2}{|c|}{ Quality }} & \multirow{3}{*}{$\begin{array}{c}\text { Quantity } \\
\text { Rate }\end{array}$} & \multirow{3}{*}{ Healthy } & \multicolumn{3}{|c|}{ Xerostomic } \\
\hline & & & & & Mild & Moderate & Severe \\
\hline & $\begin{array}{c}\text { Less than } \\
25 \mathrm{~mm}\end{array}$ & $\begin{array}{l}\text { More } \\
\text { than } \\
25 \mathrm{~mm}\end{array}$ & & & & & \\
\hline $\begin{array}{l}\text { Premenopausal (control } \\
\text { group) } \\
\mathrm{N}=40\end{array}$ & $\begin{array}{c}14 \\
(35 \%)\end{array}$ & $\begin{array}{c}26 \\
(65 \%)\end{array}$ & $2.21 \pm 25.02$ & $24(60 \%)$ & $\begin{array}{c}12 \\
(30 \%)\end{array}$ & $\begin{array}{c}4 \\
(10 \%)\end{array}$ & $\begin{array}{c}0 \\
(0 \%)\end{array}$ \\
\hline $\begin{array}{c}\text { Postmenopausal } \\
\text { (case group) } \\
\mathrm{N}=40\end{array}$ & $26(65 \%)$ & $14(35 \%)$ & $4.45 \pm 19.90$ & $\begin{array}{c}6 \\
(15 \%)\end{array}$ & $\begin{array}{c}19 \\
(47.5 \%)\end{array}$ & $\begin{array}{c}9 \\
(22.5 \%)\end{array}$ & $\begin{array}{c}6 \\
(15 \%)\end{array}$ \\
\hline Test result & $\mathrm{P}<\mathrm{c}$ & & $\mathrm{P}<0.01$ & & & & \\
\hline
\end{tabular}




\section{Discussion:}

The present study showed that in menopausal women, unstimulated salivary flow rate was lower than that in the control group $(\mathrm{P}<0.001)$, and $65 \%$ of menopausal women and $35 \%$ of premenopausal women had hyposalivation.

Changes in the oral cavity are due to aging and due to reduced estrogen in the oral mucosa. The oral mucosa is similar to the vaginal mucosa in its response to estrogen. ${ }^{(10)}$ Hormone replacement therapy (HRT) during menopause alleviates the discomfort caused by diminished circulating estrogen and progesterone hormones and prevents chronic diseases such as xerostomia. ${ }^{(10)}$

Receptors of sexual hormones have been identified in the oral mucosa and salivary glands. Estrogen can affect the oral mucosa and salivary flow directly or via the neural mechanisms depending on the level of estrogen. ${ }^{(11)}$ It has been confirmed that menopause could affect the oral mucosa and other body systems, and HRT in menopausal women can cause hypersalivation, increased salivary $\mathrm{pH}$ and buffering capacity. The exact mechanisms by which these changes are applied by estrogen are unknown; however, relief of discomfort with HRT can be indicative of the possible mechanism of action of estrogen against xerostomia. $^{(10,11)}$

The results of the present study are in line with most of the earlier studies. . $^{(1,5,12,13)}$

In 2015, Shirzaiy compared the unstimulated salivary flow rate and oral signs in postmenopausal and premenopausal women. ${ }^{(5)}$ The average unstimulated salivary flow rates in premenopausal women were higher than those in postmenopausal women $(\mathrm{P}<0.001)$, and the prevalence of xerostomia was $45 \%$ in postmenopausal and $12.5 \%$ in premenopausal women. ${ }^{(5)}$ These results confirm the results of our study.

In 2007, Borhan Mojabi and colleagues conducted a study to evaluate the unstimulated salivary flow rate and oral signs in menopausal women. ${ }^{(1)}$ The average rate of unstimulated salivary flow in premenopausal women was higher than that in menopausal subjects $(\mathrm{P}<0.01)$, which is similar to the results of the current study. Men and women were different with regards to three oral signs including xerostomia, irritation, and dysphagia upon consuming dry foods; these signs were more severe in the case group $(\mathrm{P}<0.05)$, but no other significant differences were noted between two genders. ${ }^{(1)}$

In a study conducted by Santosh and colleagues on the oral signs in postmenopausal women, xerostomia was more often met in the women with a similar age range $(\mathrm{P}=0.011){ }^{(8)}$

In 2014, a study was conducted by Saluja et al to evaluate the effect of menstruation, pregnancy, and menopause on the salivary flow rate, $\mathrm{pH}$ and gustatory function. ${ }^{(13)}$ The stimulated salivary flow rate (by chewing paraffin) was also evaluated. There was no significant statistical difference in the salivary flow rate between the groups, but the salivary $\mathrm{pH}$ and gustatory function in postmenopausal women were significantly reduced $(\mathrm{P}<0.05) .{ }^{(13)}$ The results of this study are different from those of our study, and the difference may be attributed to different methods of saliva measurement, differences in the age range of the participants and the number of samples.

In 2003, a study was conducted by Aghahosseini and Akhavan Karbasi to assess the quantity of saliva in premenopausal and menopausal women. ${ }^{(15)}$ This analytical-descriptive study was done with a cross-sectional design. 158 premenopausal and postmenopausal women (case group) and 83 men in the similar age range (control group) participated in the cited study. The age range in both groups was 50-69 years. In addition, mental status was compared in the two groups by Hospital Anxiety and Depression Scale (HADS) test, and the oral symptoms were evaluated by filling a questionnaire. Evaluation of the stimulated salivary flow rates showed that there was no significant statistical difference in the salivary rate between the two groups. However, the oral complications were significantly more frequent in the case group $(\mathrm{P}=0.002) .{ }^{(15)}$ The results of the cited study are different from those of our study, probably due to the differences in the methods of saliva measurement, differences in the age range and number of samples and differences in the number of subjects in the case and control groups.

The salivary volume in the present study was measured by the MST; however, in other studies, the stimulated and unstimulated salivary flow rates have been evaluated according to oral signs. Since the MST is more accurate and available and easy to use, and to concentrate on the efficiency 
of this new method, we only focused on saliva measurement with the MST. The advantages of this method include easy acceptance of the test strip by the patients and ease of implementation by the examiner; also, this method is less timeconsuming. ${ }^{(7,9)}$ In this study, we tried to match the age range of the case and control subjects to decrease the effect of age. In the current study, the measurement of unstimulated salivary flow rate was performed, which has a high validity. There was no bias in this research, and the results of the MST and modified Fox questionnaire confirmed the study's hypothesis.

According to the present study, menopause affects the salivary flow rate and xerostomia and can cause other oral problems such as burning mouth syndrome, increased risk of tooth decay, dysphasia, dysgeusia, atrophic gingivitis, periodontitis and jaw osteoporosis. Dentists can prevent these complications of menopause by being aware of the symptoms.

Continuous follow-up and greater use of water and other fluids, use of chewing gum and other products that can increase the salivary flow rate are recommended during menopause. Also, more attention should be paid to oral and dental hygiene during this period. Use of mucous membrane moistening agents such as Hypozalix artificial saliva, Kin Hidrat spray, and carmellose sodium $1 \% \mathrm{w} / \mathrm{v}$ can help reduce the symptoms of xerostomia. ${ }^{(14)}$ Also, brochures containing information about the oral complications of menopause along with other medical information can increase the knowledge of patients.

Conclusion

It seems that the unstimulated salivary flow rate decreases after menopause.

\section{Acknowledgements:}

Our appreciation is extended to all the patients referring to the dental branch of Tehran, Islamic Azad University who participated in this project. We are also thankful to the instructors and advisors who helped us during the culmination of this research. This study is based on student thesis \#25184 registered at the dental branch of Tehran, Islamic Azad University.

\section{References:}

1.Borhan Mojabi K, Esfahani M, Jahani Hashemi H. Evaluation of Unstimulated Salivary Flow Rate and Oral Symptoms in Menopausal Women. J Dent (Tehran) 2007;4(3):103-6.

2.Minicucci EM, Pires RB, Vieira RA, Miot HA, Sposto MR. Assessing the impact of menopause on salivary flow and xerostomia. Aust Dent J 2013;58(2):230-4.

3.Osterberg T, Landahl S, Hedegård B. Salivary flow, saliva, $\mathrm{pH}$ and buffering capacity in 70-year-old men and women. Correlation to dental health, dryness in the mouth, disease and drug treatment. J Oral Rehabil 1984;11(2):157-70.

4.Arbabi-Kalati F, Mohammadi A, Ghaemi Hesaroeeh S. Assessment the Relationship between Osteoporosis and Oral Dryness in Postmenopausal Women. J Mash Dent Sch 2015;39(2):117-22. 5.Shirzaiy M. Comparison of unstimulated salivary flow rate and oral symptoms between premenopausal and postmenopausal women. J Qazvin Univ Med Sci 2015;19(2):39-45.

6. Bowers L, Fox P, Brennan M. Hyposalivation, the salivary gland disease. In: Burket's Oral Medicine. 12th ed. House-USA Shelton 2015:251252.

7.Dyasanoor S, Saddu SC. Association of Xerostomia and Assessment of Salivary Flow Using Modified Schirmer Test among Smokers and Healthy Individuals: A Preliminutesary Study. J Clin Diagn Res 2014 Jan;8(1):211-3.

8.Santosh P, Nidhi S, Sumita K, Farzan R, Bharati D, Ashok K. Oral findings in postmenopausal women attending dental hospital in Western part of India. J Clin Exp Dent 2013;5(1):e8-e12.

9.Chen A, Wai Y, Lee L, Lake S, Woo SB. Using the modified Schirmer test to measure mouth dryness: a preliminary study. J Am Dent Assoc 2005;136(2):164-70.

10.Suri V, Suri V. Menopause and oral health. J Midlife Health 2014;5(3):115-120.

11.Valimaa H, Savolainen S, Soukka T, Silvoniemi P, Makela S, Kujari H, et al. Estrogen receptor-beta is the predominant estrogen receptor subtype in human oral epithelium and salivary glands. J Endocrinol 2004;180(1):55-62.

12.Konttinen YT, Stegaev V, Mackiewicz Z, Porola P, Hänninen A, Szodoray P. Salivary glands "an unisex organ"? Oral Dis 2010;16(7):577-85. 
13.Saluja P, Shetty V, Dave A, Arora M, Hans V, Madan A. Comparative Evaluation of the Effect of Menstruation, Pregnancy and Menopause on Salivary Flow Rate, $\mathrm{pH}$ and Gustatory Function. J Clin Diagn Res 2014;8(10):ZC81-5.

14.Jose A, Siddiqi M, Cronin M, DiLauro TS, Bosma ML. A randomized clinical trial in subjects with dry mouth evaluating subjective perceptions of an experimental oral gel, an oral rinse and a mouth spray compared to water. Am J Dent 2016;29(1):58-64.

15.Aghahosseini F, Akhavan Karbasi MH. An investigation on the stimulated salivary flow rate in menopause and post menopause women. J Dent Med 2003;16(2):39-45. 